\title{
Active plasmonic devices and optical metamaterials
}

\author{
Koray Aydin ${ }^{1}$, Stanley Burgos ${ }^{1}$, Imogen M. Pryce ${ }^{1}$, Matthew J. Dicken ${ }^{1}$, Jennifer A. Dionne ${ }^{1}$, Kenneth \\ Diest $^{1}$, Rene de Waele ${ }^{2}$, Albert Polman ${ }^{2}$, Harry. A. Atwater ${ }^{1}$ \\ ${ }^{1}$ Thomas J. Watson Lab. of Applied Physics, California Institute of Technology, Pasadena, CA 91125. \\ ${ }^{2}$ FOM Institute for Atomic and Molecular Physics([AMOLF), Amsterdam, Netherlands.
}

\begin{abstract}
We studied active near-infrared metamaterials based on phase transition of vanadium oxide thin films, asymmetrically coupled split-ring resonators for narrowing resonance line-widths, field effect modulation of plasmon propagation and 3D single layer, plasmonic negative-index metamaterials.
\end{abstract}

\section{Frequency Tunable Infrared Metamaterials}

We present the demonstration of frequency tunable infrared metamaterials whose basic elements are $\mathrm{Ag} / \mathrm{VO}_{2}$ hybrid split ring resonators (SRR). Vanadium oxide $\left(\mathrm{VO}_{2}\right)$ is a promising phase-transition material that can be used in designing active metamaterials, which undergoes a phase transition from a semiconducting monoclinic phase to a metallic rutile phase at $68{ }^{\circ} \mathrm{C}$. Thin films of vanadium dioxide have been grown by pulsed laser deposition on sapphire substrate. The complex refractive indices of thin films in both phases were determined by spectroscopic ellipsometry. The split ring resonator frequency response was determined using full field electromagnetic simulations for bilayer $\mathrm{Ag}-\mathrm{VO}_{2}$ split ring resonator

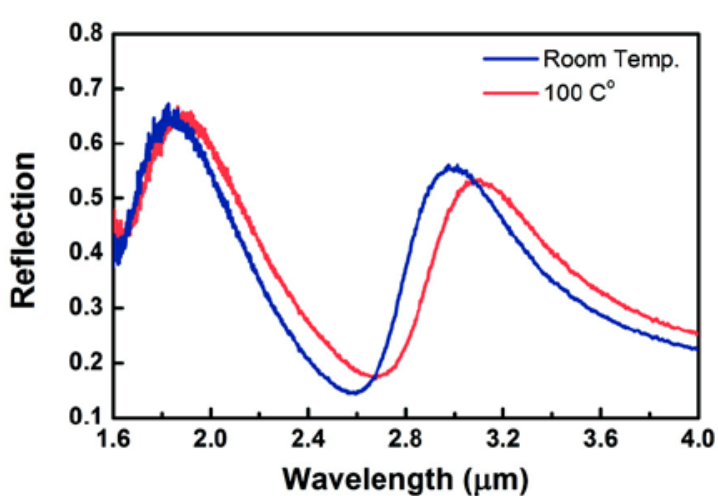

Fig. 1. Resonant peak shift of $110 \mathrm{~nm}$ is observed as the self-aligned $\mathrm{Ag} / \mathrm{VO}_{2}$ hybrid metamaterial is thermally switched above the $\mathrm{VO}_{2}$ insulator-metal phase transition. (SRR) arrays with complex refractive index models for the semiconductor and metallic phases of vanadium oxide. $\mathrm{Ag}$ SRR arrays were patterned on $60 \mathrm{~nm}$ thick $\mathrm{VO}_{2}$ film using electron-beam lithography. Using infrared spectroscopy, we have observed suppression of the resonant reflection peak at $3.2 \mu \mathrm{m}$ by thermal actuation of $\mathrm{VO}_{2}$ thin films. $\mathrm{VO}_{2}$ thin films were then etched to form bi-layer $\mathrm{Ag}-\mathrm{VO}_{2}$ hybrid SRR arrays. Full-field electromagnetic simulations have predicted that bi-layer hybrid SRR design yields a highly tunable frequency response upon thermal actuation of the $\mathrm{VO}_{2}$ layer. Self-aligned, hybrid $\mathrm{Ag} / \mathrm{VO}_{2}$ laminate structures allow us to demonstrate optical and geometrical engineering of metamaterial devices leading to resonant peak position tuning of $110 \mathrm{~nm}$ at near-IR wavelengths (Fig. 1).

\section{Asymmetric Split Ring Resonators}

We studied the effect of coupled asymmetric split-ring resonators for reducing the spectral linewidth at the resonance frequency. A narrower resonant peak will increase the tuning figure of merit (FOM), the ratio of the tuning range to the full width at half maximum (FWHM) of the resonant peak. Figure 2 shows the numerical simulations of asymmetric SRRs that are coupled face-to-face with different 
spacings (inset of Fig. 2(b)). Simply, by breaking the structural symmetry of metamaterials we have shown narrower metamaterial resonances could be obtained. We fabricated arrays of Au SRRs on ITO coated glass substrates and experimental measurements are performed at the near-IR wavelengths using FTIR. Fullfield electromagnetic simulations with Lumerical, agree well with the experimental results. Metamaterial resonances can be engineered using different coupling and hybridization mechanisms. Different geometries and coupling schemes were investigated by means of experiments and numerical simulations that show transmission resonances of different magnitudes, at wavelengths dependant on the size and degree of coupling between SRRs. We have calculated the magnetic and electric field intensities inside SRR structure that provided insights on the differences of various coupling mechanisms.

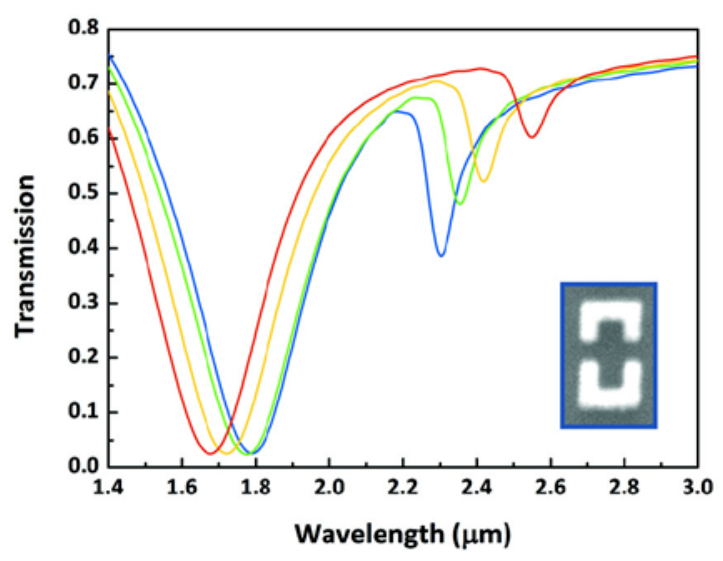

Fig. 2. Calculated transmission spectra of different face-toface coupled SRRs with varying separation. Unit cell of a fabricated device is shown in the inset.

\section{Three Dimensional All-Angle Negative Index Metamaterials}

We expand upon recently reported work on direct observation of two-dimensional negative refraction in the visible frequency range to develop a general approach to realization of three-dimensional single-layer,

all-angle,

independent

metamaterials negative refraction. The single layer negative index material is composed of an array of vertically-oriented coaxial metal-insulator-metal structures. Full wave simulations such as those shown in Fig. 3 and dispersion calculations are used to demonstrate that metaldielectric-metal plasmonic waveguides are characterized by negative wave vectors and negative refractive indices. We also have been able to show that thin wedge-shaped metamaterial sheets composed of arrays of coaxial metal-dielectric-metal plasmonic structures exhibit negative refractive for transmitted beams projected into the far field.

\section{Active Plasmonic Devices - PlasMOStor}

Metal-dielectric plasmon waveguides can serve as active switching elements when the dielectric refractive index can be actively modulated. We demonstrate electro-optic refractive index modulation in metaldielectric-metal plasmon waveguides using low-voltage electro-optic modulation of both silicon metaloxide-semiconductor plasmonic resonators to yield a "plasMOStor" - an MOS field effect plasmonic modulator-with $11 \mathrm{~dB}$ on/off ratio in a footprint of a few square microns. 